\title{
Estudo de mecanismos e fatores relacionados com o abuso sexual em crianças e adolescentes do sexo feminino
}

\section{Study of mechanisms and factors related to sexual abuse in female children and adolescents}

\author{
Jefferson Drezett ${ }^{1}$, Marcelo Caballero ${ }^{2}$, Yara Juliano ${ }^{3}$, Elizabeth T. Prieto ${ }^{4}$, \\ José A. Marques ${ }^{5}$, César E. Fernandes 6
}

\section{Resumo}

Objetivo: estudar mecanismos e fatores relacionados com o abuso sexual, comparando suas freqüências entre vítimas crianças e adolescentes.

Métodos: estudo retrospectivo de 617 vítimas de abuso sexual atendidas entre julho de 1994 e agosto de 1999 pelo Centro de Referência da Saúde da Mulher; 71 crianças (idade < 10 anos) e 546 adolescentes (10 a 20 anos). As variáveis de estudo foram crime sexual; constrangimento; presunção de violência; tipificação do agressor; número de agressores; situação da vítima no momento do crime; e ocorrência de traumas físicos. Os dados foram informatizados em programa Epi Info 6 e os resultados analisados pelo teste de $\mathrm{C}^{2}$.

Resultados: o estupro ocorreu em $90,8 \%$ das adolescentes e o atentado violento ao pudor em $46,5 \%$ das crianças. A violência presumida (VP) foi mais freqüente nas crianças $(63,4 \%)$ e a grave ameaça nas adolescentes $(63,2 \%)$. A inocencia consilli foi VP exclusiva entre crianças, atingindo 59,5\% das adolescentes. Entre crianças, $84,5 \%$ foram abusadas por agressores identificáveis, geralmente do núcleo familiar, enquanto desconhecidos violentaram $72,3 \%$ das adolescentes. Nas crianças, o abuso ocorreu em 42,3\% em suas residências e em $28,2 \%$ na do agressor. Adolescentes foram vitimadas durante atividades cotidianas $(34,8 \%)$ e no percurso do trabalho ou escola $(28,4 \%)$. A maioria das pacientes não apresentou traumas físicos genitais ou extragenitais.

Conclusões: crianças foram submetidas, principalmente, ao atentado violento ao pudor, perpetrado por agressor conhecido, por meio de violência presumida e em ambientes privados. Nas adolescentes predominou o estupro por agressores desconhecidos, sob grave ameaça, em ambientes não domésticos.

J Pediatr (Rio J) 2001; 77 (5): 413-9: maus-tratos sexuais infantis, violência, estupro.

1. Doutor em Ginecologia e Coord. do Serviço de Atenção Integral à Mulher Sexualmente Vitimada do Centro de Referência da Saúde da Mulher, São Paulo, SP.

2. Ginecologista assistente do Serviço de Atenção Integral à Mulher Sexualmente Vitimada e Responsável pelo Serviço de DST/Aids do Centro de Referência da Saúde da Mulher.

3. Professora Titular da Disciplina de Medicina Coletiva da Universidade de Santo Amaro.

4. Ginecologista Estagiária do Centro de Referência da Saúde da Mulher.

5. Doutor em Ginecologia. Diretor do Departamento do Núcleo da Saúde da Mulher do Centro de Referência da Saúde da Mulher.

6. Doutor em Tocoginecologia. Diretor Científico do Centro de Referência da Saúde da Mulher.

\section{Abstract}

Objective: to study the frequency of sexual abuse in children and adolescents and its related factors.

Methods: retrospective analysis of 617 cases of sexual abuse, assisted between July 1994 and August 1999 at the Women's Health Reference Center, divided into two groups: 71 children (age $<10$ years), and 546 adolescents (age $>$ or $=10$ and $<20$ years). The analyzed variables were: sexual crime; embarrassment; presumption of violence; characteristics and number of abusers; the victim's situation at the moment of the crime; and occurrence of physical traumas. Epi Info 6 software was utilized to gather data, and the results were analyzed by the chi-square test.

Results: significant statistic results were found. $90.8 \%$ of the adolescents were victims of rape, and $46.5 \%$ of the children suffered sexual assault. The presupposed violence (PV) was more frequent in the group of children $(63.4 \%)$ and a serious threat in the group of adolescents $(63.2 \%)$. Innocentia consilli was exclusive PV among $100 \%$ of children and $59 . .5 \%$ of adolescents. $84.5 \%$ of children were molested by identifiable abusers, most frequently family members. Perpetrators were unknown in $72.3 \%$ of the cases of adolescent abuse. $42.3 \%$ of sexual abuse of children occurred in their homes, and $28.2 \%$ at the abuser's. Adolescents were approached during daily activities (34.8\%) and on their way to work or school (28.4\%). Most patients did not have genital or extragenital trauma.

Conclusions: children suffered sexual assault, perpetrated by a known abuser through presupposed violence in private places. Adolescents were raped by unknown individuals, under serious threat, in nondomestic places.

J Pediatr (Rio J) 2001; 77 (5): 413-9: child abuse, violence, rape.

\section{Introdução}

O abuso sexual é fenômeno universal que atinge, indistintamente, todas as classes sociais, etnias, religiões e culturas. Sua verdadeira incidência é desconhecida, acreditando-se ser uma das condições de maior subnotificação e subregistro em todo o mundo ${ }^{1}$. Apesar do tímido percentual de denúncias, a violência sexual é cada vez mais reportada, estimando-se que acometa 12 milhões de pessoas, a cada ano, em todo o mundo. Apenas nos EUA, calcula-se que 
ocorra uma agressão sexual a cada 6,4 minutos e que uma em cada quatro mulheres tenha sofrido algum contato sexual não consentido durante a infância ou adolescência. Entre crianças, estima-se que um terço tenha sido submetido, alguma vez, a um contato incestuoso. Atualmente, cerca de 200 mil crianças americanas são sexualmente abusadas, a cada ano $^{2}$.

Devido a sua elevada incidência e prevalência, bem como aos danos e agravos que determinam, os crimes sexuais adquiriram proporções de complexo problema de saúde pública. Não obstante, deve-se acrescer que o abuso sexual apresenta estreita interface com questões policiais e jurídicas, exigindo do médico e da equipe interdisciplinar conhecimentos apropriados de medicina legal e sexologia forense ${ }^{1}$.

Nesse sentido, o Código Penal Brasileiro define como estupro o constrangimento de pessoas do sexo feminino ao coito vaginal, mediante violência ou grave ameaça. Entende-se por "violência" o emprego de força física capaz de sobrepujar a força da vítima; e por "grave ameaça" a promessa de efetuar tamanho mal, suficiente para impedir sua resistência. $\mathrm{O}$ atentado violento ao pudor é caracterizado pelo constrangimento de pessoas de ambos os sexos, mediante violência e/ou grave ameaça, à pratica de atos libidinosos diferentes do coito vaginal ${ }^{3}$.

Em condições excepcionais, mesmo não ocorrendo o uso de força ou de ameaça, pode-se igualmente caracterizar o crime sexual. Essas situações, denominadas "violência presumida", incluem pessoas menores de 14 anos; deficientes mentais; ou aquelas que não podem, por qualquer outra causa, oferecer resistência ${ }^{2}$. O limite de idade de 14 anos é fundamentado, legalmente, na condição de inocencia consilli, traduzida pela completa falta de ciência em relação aos fatos sexuais ${ }^{4}$.

Considerando-se os múltiplos aspectos envolvidos com a violência sexual, existe ainda grande desconhecimento dos mecanismos e consequiências da violência, principalmente em nosso meio. O presente artigo tem por objetivo contribuir com a questão, estudando alguns fatores relacionados com o abuso sexual entre crianças e adolescentes do sexo feminino.

\section{Métodos}

Os dados foram coletados de 617 crianças e adolescentes do sexo feminino, sexualmente abusadas, matriculadas no Centro de Referência da Saúde da Mulher e de Nutrição, Alimentação e Desenvolvimento Infantil (CRSMNADI), entre julho de 1994 e agosto de 1999. Quanto à origem dos encaminhamentos, 258 casos $(41,8 \%)$ foram enviados pelas Delegacias de Polícia; 189 casos (30,6\%) pelo Instituto Médico Legal (IML); 87 (14,1\%) por outros serviços de saúde; e 17 (2,7\%) pelo Poder Judiciário. Em 66 casos $(10,6 \%)$ a procura foi espontânea.
As pacientes foram alocadas em dois grupos de estudo, definidos pela idade no momento do crime sexual, segundo critério da Organização Mundial da Saúde: crianças (idade inferior a dez anos completos) e adolescentes (idade maior ou igual a dez anos completos e menor que 20 anos) $)^{5}$.

Foram considerados critérios de inclusão os crimes sexuais de estupro; atentado violento ao pudor anal (AVPA); atentado violento ao pudor oral (AVPO); e atentado violento ao pudor (AVP) diferente do AVPA e do AVPO. Para a caracterização desses crimes sexuais foi adotada a definição dos artigos 213 e 214 do Código Penal Brasileiro 3 .

Foram excluídos casos de posse sexual mediante fraude; atentado ao pudor mediante fraude; sedução; corrupção de menores; casos envolvendo o sexo masculino; crimes de caracterização duvidosa; casos de falsa alegação e tentativas de crime sexual não consumadas.

As variáveis de estudo foram analisadas considerandose apenas as categorias de inclusão, como se seguem: A) crime sexual - classificado em uma das sete categorias: estupro, AVPA, AVPO, AVP diferente do AVPO e AVPA, estupro e AVPA, estupro e AVPO, e estupro e AVPA e AVPO; B) forma de constrangimento - classificado em uma das quatro categorias: força física, grave ameaça, associação de força física e de grave ameaça, e violência presumida; C) presunção da violência - em uma das quatro categorias: deficiência mental, uso de substâncias hipnóticas, inocencia consilli, e embriaguez; D) agressor - tipificado em uma das 11 categorias: desconhecido, pai biológico, padrasto, tio, avô, irmão, vizinho, ex-parceiro, atual parceiro, conhecido do trabalho, e outros conhecidos; E) número de agressores - quantificação dos agressores em uma de duas categorias: único e múltiplo; F) atividade da vítima classificada em uma das sete categorias: proximidade da residência, percurso da escola ou trabalho, local de trabalho, residência da vítima, residência do agressor, relacionada com lazer, e ignorado; G) trauma genital - em uma das cinco categorias: sem trauma, hematoma de vulva, fissura de fúrcula, fissura perianal, e rotura de períneo; H) trauma extragenital - em uma das sete categorias: sem trauma, hematoma, escoriação, mordedura, ferimento corto-contuso, queimadura, e outro tipo de trauma.

Para a coleta de dados, utilizaram-se as informações prestadas pela vítima e/ou seu representante, registradas consensualmente pelo ginecologista, psicólogo e assistente social. As entrevistas foram registradas utilizado-se de ficha de atendimento interdisciplinar padronizada e précodificada. Os dados foram introduzidos em programa Epi Info 6, versão 6.04b. A verificação da consistência dos dados foi realizada por meio de dupla digitação, comparação dos arquivos pelo Epi Info 6 e correção de divergências.

Para a análise estatística foi aplicado o teste de qui quadrado $\left(c^{2}\right)$ para tabelas de contingência e de associação, fixando-se em $0,05(\mathrm{p} \leq 0,05)$ o nível de rejeição da hipótese de nulidade, assinalando-se com um asterisco $(*)$ os valores significantes $^{6}$. 
O Projeto de Pesquisa foi submetido à apreciação do Comitê de Ética em Pesquisa do CRSMNADI, sob n ${ }^{\circ} 027 /$ 99, e aprovado em 17 de novembro de 1999.

\section{Resultados}

A idade variou entre 1 e 19 anos, com média de 5,8 anos para as crianças e de 15,6 anos para as adolescentes. A Tabela 1 mostra a distribuição dos crimes sexuais, onde se observa a predominância do crime de estupro que, de forma isolada ou associada com outros crimes, ocorreu em 83,7\% dos casos. O grupo de crianças apresentou frequiência de "outro tipo de AVP" 13 vezes maior que as adolescentes, resultando em diferença estatisticamente significante.

$\mathrm{Na}$ Tabela 2, forma de constrangimento utilizada pelo agressor, constatou-se que a violência presumida foi mais freqüente nas crianças, quase nove vezes maior do que nas adolescentes. Por outro lado, o constrangimento mediante o uso da grave ameaça apresentou maior freqüência no grupo de adolescentes, diferença considerada estatisticamente significante.

Na Tabela 3, encontra-se a distribuição de 82 casos de violência presumida. A condição de inocencia consilli foi o achado mais freqüente, 4,4 vezes maior que a soma das demais categorias. Entre as crianças, a inocencia consilli foi condição absoluta, atingindo 59,5\% das adolescentes.

O perpetrador foi apontado como desconhecido por $72,3 \%$ das adolescentes (395 casos) e por $15,5 \%$ das crianças (11 casos), resultando $\mathrm{c}^{2}$ calculado de 90,24 $(p<0,001)$, diferença estatisticamente significativa. A tipificação dos agressores identificados encontra-se na Tabela 4. A somatória dos agressores do núcleo familiar ou aparentados da vítima apresentou freqüência elevada em ambos os grupos estudados, sendo maior no grupo de crianças $(60,0 \%)$ do que entre adolescentes $(38,5 \%)$. Como categoria isolada, moradores da vizinhança da vítima atingiram as maiores freqüências, responsáveis por cerca de um quarto dos casos de abuso sexual em cada grupo.

$\mathrm{O}$ agressor único prevaleceu nos dois grupos, em mais de $90 \%$ dos casos. Nas crianças, apenas um caso $(1,4 \%)$ foi consumado por dois perpetradores. Em 45 adolescentes $(8,3 \%)$, houve mais do que um agressor, variando de dois a seis elementos.

A Tabela 5 apresenta a distribuição da atividade das vítimas no momento da abordagem do agressor. Em 63,2\% das adolescentes o crime ocorreu durante o percurso do trabalho ou escola, ou durante atividades cotidianas realizadas na proximidade de sua residência. Entre as crianças foi maior a freqüência de casos dentro de sua residência $(42,3 \%)$ ou do agressor $(28,2 \%)$. Crimes praticados durante atividades de lazer foram dez vezes mais freqüentes nas adolescentes $(15 \%)$ do que nas crianças $(1,4 \%)$.

A ocorrência de trauma genital foi calculada considerando-se apenas casos de estupro e/ou AVPA envolvendo 34 crianças e 520 adolescentes. Lesões genitais foram seis vezes mais freqüentes em crianças $(17,6 \%)$ do que em adolescentes $(2,7 \%)$. Nas crianças predominou a fissura perianal $(8,8 \%)$, freqüência onze vezes maior do que nas adolescentes $(0,8 \%)$; e a rotura perineal $(8,8 \%)$, freqüência 44 vezes maior do que nas adolescentes $(0,2 \%)$. Entre adolescentes o trauma genital mais freqüente foi a fissura de fúrcula vaginal $(0,9 \%)$.

A ocorrência de trauma extragenital foi observada em 60 adolescentes (11\%), sendo o hematoma o achado de maior frequiência $(3,5 \%)$, seguido de escoriações de diferentes extensões $(3,1)$. Entre crianças não observou-se a ocorrência de traumas extragenitais.

Tabela 1 - Tipo de crime sexual perpetrado, consoante as faixas etárias estudadas

\begin{tabular}{lcccccc}
\hline & \multicolumn{2}{c}{ Crianças } & \multicolumn{2}{c}{ Adolescentes } & \multicolumn{2}{c}{ Total } \\
Tipo de crime sexual & $\mathbf{n}$ & $\mathbf{\%}$ & $\mathbf{n}$ & $\boldsymbol{\%}$ & $\mathbf{n}$ & $\%$ \\
\hline Estupro & 12 & 16,9 & 323 & $59,2^{*}$ & 335 & 54,3 \\
Estupro + AVPA & 5 & 7,0 & 83 & 15,2 & 88 & 14,2 \\
Estupro + AVPO & 3 & 4,2 & 51 & 9,3 & 54 & 8,7 \\
Estupro + AVPA + AVPO & 1 & 1,4 & 39 & 7,1 & 40 & 6,5 \\
AVPA & 13 & 18,3 & 24 & 4,4 & 37 & 6,0 \\
AVPO & 4 & 5,6 & 7 & 1,3 & 11 & 1,9 \\
Outro tipo de AVP & 33 & $46,5 *$ & 19 & 3,5 & 52 & 8,4 \\
Total & 71 & 100 & 546 & 100 & 617 & 100 \\
& & & & & &
\end{tabular}

Teste de $c^{2} \quad c^{2}$ calculado $=193,45^{\star} \quad(p<0,001) \quad c^{2}$ crítico $=12,59$

AVPA: atentado violento ao pudor com coito anal

AVPO: atentado violento ao pudor com coito oral

AVP: atentado violento ao pudor diferente do AVPA e do AVPO 
Tabela 2 - Distribuição da forma de constrangimento utilizada para a perpetração do crime sexual

\begin{tabular}{|c|c|c|c|c|c|c|}
\hline \multirow[b]{2}{*}{ Constrangimento } & \multicolumn{2}{|c|}{ Crianças } & \multicolumn{2}{|c|}{ Adolescentes } & \multicolumn{2}{|c|}{ Total } \\
\hline & $\mathbf{n}$ & $\%$ & $\mathbf{n}$ & $\%$ & $\mathbf{n}$ & $\%$ \\
\hline Grave ameaça (GA) & 16 & 22,5 & 345 & $63,2 *$ & 361 & 58,6 \\
\hline $\mathrm{FF}+\mathrm{GA}$ & 5 & 7,0 & 97 & 17,8 & 102 & 16,5 \\
\hline Força física (FF) & 5 & 7,0 & 67 & 12,3 & 72 & 11,6 \\
\hline Violência presumida & 45 & $63,4^{*}$ & 37 & 6,8 & 82 & 13,3 \\
\hline Total & 71 & 100 & 546 & 100 & 617 & 100 \\
\hline
\end{tabular}

\section{Discussão}

Apesar da violência sexual ocorrer em qualquer idade, a maioria dos registros aponta para a predominância desses crimes entre as mais jovens e adolescentes ${ }^{7-16}$. Não restam dúvidas quanto ao impacto que o abuso sexual acarreta para a vítima. Quando ocorrido na infância, pode resultar em maior risco de prostituição na vida adulta e de gravidez na adolescência ${ }^{2,11}$

O médico deveria estar preparado para o manejo clínico e psicológico dessas vítimas. $\mathrm{O}$ atendimento exige, além de treinamento e capacitação, paciência e experiência. A negligência para com esses aspectos pode resultar na revitimização da paciente pelos serviços de saúde $7,17,18$.

Neste estudo, para análise do tipo de crime sexual, optamos por comparar os resultados com outros verificados em nosso meio, dada a diversidade de terminologia e de classificação desses crimes pelas legislações de outros países ${ }^{19}$. No entanto, a análise dessa variável sofreu significativa modificação em função da idade. Enquanto nas adolescentes predominou o estupro isolado ou associado ao AVP $(90,8 \%)$, entre as crianças prevaleceu o AVP diferente do AVPO e AVPA (46,5\%), resultando em diferença esta- tisticamente significante (Tabela 1). Destaque deve ser dado ao AVPA entre as crianças (18,3\%), que apresentou freqüência similar àquela encontrada para o estupro (16,9\%). Dados da ABRAPIA (1997) revelam grande similaridade com os deste estudo, com cerca de $70 \%$ das crianças submetidas ao AVP e $10 \%$ ao estupro consumado ${ }^{8}$.

Acredita-se que a força física do agressor e a intimidação psicológica por ele aplicada sejam fatores determinantes para neutralizar a resistência da vítima ${ }^{9,19}$. Neste estudo, a intimidação psicológica foi a forma predominante de constrangimento $(58,6 \%)$ entre as pacientes analisadas (Tabela 2), constatação observada por diversos autores $^{9,15,19}$. No entanto, a grave ameaça foi mais freqüente nas adolescentes $(63,2 \%)$ do que nas crianças $(22,5 \%)$, resultando em diferença estatisticamente significativa. Em 82 casos $(13,3 \%)$ verificou-se a presunção de violência. Nas crianças, encontrou-se a condição de inocencia consilli como único fator de violência presumida, enquanto que nas adolescentes foi expressivo o número de casos de deficiência mental $(35,1 \%)$. De fato, estima-se que cerca de metade das deficientes mentais são sexualmente agredidas, pelo menos uma vez, ao longo de suas vidas ${ }^{20}$.

Tabela 3 - Distribuição do tipo de violência presumida em 82 pacientes, consoante as faixas etárias estudadas

\begin{tabular}{lcccccc}
\hline \multirow{2}{*}{ Violência presumida } & \multicolumn{2}{c}{ Crianças } & \multicolumn{2}{c}{ Adolescentes } & \multicolumn{2}{c}{ Total } \\
& $\mathbf{n}$ & $\mathbf{\%}$ & $\mathbf{n}$ & $\mathbf{\%}$ & $\mathbf{n}$ & $\%$ \\
\hline Inocencia consilli & 45 & 100 & 22 & 59,5 & 67 & 81,7 \\
Deficiência mental & 0 & 0 & 13 & 35,1 & 13 & 15,9 \\
Uso de hipnóticos & 0 & 0 & 1 & 2,7 & 1 & 1,2 \\
Embriaguez & 0 & 0 & 1 & 2,7 & 1 & 1,2 \\
Total & 45 & 0 & 37 & 100 & 82 & 100 \\
\hline
\end{tabular}


Tabela 4 - Tipificação de 211 agressores identificados como responsáveis pela perpetração do crime sexual, consoante as faixas etárias estudadas

\begin{tabular}{lcccccc}
\hline \multirow{2}{*}{ Agressor } & \multicolumn{2}{c}{ Crianças } & \multicolumn{2}{c}{ Adolescentes } & \multicolumn{2}{c}{ Total } \\
& $\mathbf{n}$ & $\mathbf{\%}$ & $\mathbf{n}$ & $\mathbf{\%}$ & $\mathbf{n}$ & $\%$ \\
\hline Pai biológico & 13 & 21,7 & 21 & 13,9 & 34 & 16,1 \\
Padrasto & 10 & 16,7 & 16 & 10,6 & 26 & 12,3 \\
Tio & 7 & 11,6 & 14 & 9,3 & 21 & 9,9 \\
Avô & 6 & 10,0 & 0 & 0 & 6 & 2,8 \\
Irmão & 0 & 0 & 7 & 4,7 & 7 & 3,3 \\
Vizinho & 10 & 16,7 & 42 & 27,8 & 52 & 24,7 \\
Ex-parceiro & - & - & 9 & 5,9 & 9 & 4,3 \\
Parceiro atual & - & - & 4 & 2,6 & 4 & 1,9 \\
Conhecido do trabalho & - & - & 8 & 5,3 & 8 & 3,8 \\
Outro conhecido & 14 & 23,3 & 30 & 19,9 & 44 & 20,9 \\
Total & 60 & 100 & 151 & 100 & 211 & 100 \\
\hline
\end{tabular}

(-) não aplicável

Neste estudo, foi pouco expressiva a alegação do uso de substâncias hipnóticas como forma de impedimento de resistência da vítima. Essas situações, de escasso registro em nosso meio, tem assumido destacada importância nos EUA, onde mais de 20 substâncias diferentes foram identificadas para esse fim $^{21}$.

$\mathrm{O}$ agressor desconhecido associou-se às adolescentes $(72,3 \%)$, enquanto a maioria das crianças $(84,5 \%)$ pôde identificar o agressor. Resultados heterogêneos são descritos pela literatura. Para alguns autores, o agressor foi identificado em cerca de $70 \%$ dos casos entre adolescentes e adultas jovens. Para outros, o desconhecido assume freqüência entre 50 e $70 \%{ }^{15,21-23}$. Quanto à elevada ocorrência de agressores conhecidos entre as crianças, nossos resultados foram semelhantes à maioria dos apontamen$\operatorname{tos}^{16,24}$.
A tipificação do agressor conhecido apresentou importante variação nos dois grupos etários (Tabela 4). Segundo a ABRAPIA (1997), no abuso sexual contra crianças e adolescentes, o pai biológico representa cerca de $30 \%$ dos casos; o avô $2 \%$; o padrasto $12 \%$; vizinho $8 \%$; irmão $1 \%$; e tio $4 \%{ }^{8}$. Neste estudo, resultados semelhantes foram verificados quanto à agressão pelo pai biológico e padrasto. No entanto, observamos percentuais mais expressivos para o avô entre as crianças (10\%), irmão entre as adolescentes $(4,6 \%)$, e tio para ambos os grupos $(11,6 \%$ e $9,4 \%$, respectivamente). Entre os agressores sem laços consangüíneos com a paciente, um vizinho foi o elemento mais freqüente nos dois grupos. A maioria das pacientes deste estudo foram vitimadas por agressor único. Há dados consistentes que apontam seqüelas psicológicas mais severas quando o abuso sexual é perpetrado por múltiplos perpetradores, principalmente durante a infância e a adolescência ${ }^{25}$.

Tabela 5 - Atividade ou situação da vítima no momento da abordagem do agressor, consoante as faixas etárias estudadas

\begin{tabular}{lcccccc}
\hline & \multicolumn{2}{c}{ Crianças } & \multicolumn{2}{c}{ Adolescentes } & \multicolumn{2}{c}{ Total } \\
Atividade / Situação & $\mathbf{n}$ & $\mathbf{\%}$ & $\mathbf{n}$ & $\mathbf{\%}$ & $\mathbf{n}$ & $\%$ \\
\hline Próxima à residência & 16 & 22,5 & 190 & 34,8 & 206 & 33,4 \\
Percurso trabalho/escola & 3 & 4,2 & 155 & 28,4 & 158 & 25,7 \\
Residência da vítima & 30 & 42,3 & 83 & 15,2 & 113 & 18,3 \\
Relacionada com lazer & 1 & 1,4 & 82 & 15,0 & 83 & 13,4 \\
Residência do agressor & 20 & 28,2 & 23 & 4,2 & 43 & 6,9 \\
Local de trabalho & - & - & 10 & 1,8 & 10 & 1,7 \\
Ignorado & 1 & 1,4 & 3 & 0,6 & 4 & 0,6 \\
Total & 71 & 100 & 546 & 100 & 617 & 100 \\
\hline
\end{tabular}

(-) não aplicável 
Quanto à atividade da vítima no momento da abordagem do agressor (Tabela 5), verificou-se que as ocorrências em espaços públicos estiveram relacionadas, principalmente, com as adolescentes $(78,2 \%)$. Nas crianças, constatou-se que a maioria dos crimes sexuais transcorreu em ambiente privado $(70,5 \%)$, principalmente na residência $(42,3 \%)$. Os resultados deste estudo estão de acordo com os da literatura, que aponta o abuso sexual na infância como fenômeno predominantemente intra-familiar ${ }^{26}$.

Assim como os de outros autores, nossos resultados quanto à ocorrência de trauma físico mostraram baixa taxa de lesões genitais e extragenitais. Contudo, entre as crianças estudadas, além da freqüência seis vezes maior de danos genitais, verificou-se que os traumas mais severos, como a rotura perineal, obtiveram freqüência 40 vezes maior. A menor frequiência de trauma genital entre adolescentes $(2,7 \%)$ pode estar relacionada, entre outros fatores, com o maior desenvolvimento biológico e início da vida sexual antes da agressão. Por outro lado, é possível que a maior ocorrência dos extragenitais nesse grupo (11\%) possa advir da maior resistência oferecida ao agressor ${ }^{1}$.

Poucos estudos têm avaliado danos genitais entre vítimas que não haviam iniciado vida sexual. Contudo, parece que a prevalência dessas lesões é duas a três vezes maior nessas mulheres. Além disso, cogita-se que a rotura himenal possa aumentar significativamente o risco para DST/Aids ${ }^{1}$.

O abuso sexual na infância e na adolescência é envolvido por um profundo pacto de silêncio, principal responsável pelo tímido número de denúncias. A casuística apresentada neste estudo, apesar de expressiva, reflete, seguramente, apenas uma pequena parcela dos casos que realmente ocorrem. Sem perspectivas de romper com o processo de silêncio, a vítima depende, muitas vezes, da iniciativa de educadores, vizinhos ou amigos. Segundo o Estatuto da Criança e do Adolescente, os profissionais de saúde têm o dever de denunciar os casos suspeitos ou confirmados de que tenham conhecimento, fundamentado-se em evidências consistentes e sustentáveis ${ }^{27}$.

A exemplo de muitas violências antigas na história humana, o estupro é severamente condenado pelos textos do direito clássico, mas pouco penalizado pela justiça. A expectativa de contar com provas materiais é freqüentemente frustrada e, muitas vezes, entendida como falsa alegação. Paradoxalmente, a sociedade tem exigido que, além de violentada, a vítima apresente-se brutalmente ferida. Quando crianças, sequer admitimos que sua palavra possa ter a mesma credibilidade daquela que oferecemos ao agressor. Tendemos a considerar seus relatos fantasiosos e incapazes de diferenciar o lúdico do real, protegendo, incompreensivelmente, o perpetrador.

Entendemos que a mudança dessa realidade passa, necessariamente, pela modificação nas relações de gênero e pela transformação de nossas atitudes. Ou então, como as denaides da mitologia grega, as vítimas continuarão condenadas a carregar os seus direitos em um jarro furado.
Considerando as crianças e as adolescentes estudadas, os resultados da presente investigação permitem concluir que 1. o estupro foi o crime sexual predominante entre adolescentes, enquanto o atentado violento ao pudor foi mais freqüente entre crianças; 2. a grave ameaça, nas adolescentes, e a violência presumida, nas crianças, foram os tipos de constrangimento mais impostos; 3 . nas adolescentes foi expressiva a condição de deficiência mental nos casos de presunção de violência, enquanto que, nas crianças, a inocencia consilli foi fator exclusivo; 4 . o agressor desconhecido prevaleceu entre adolescentes, enquanto que nas crianças predominou o agressor identificável, principalmente os do núcleo familiar; 6. adolescentes foram geralmente vitimadas durante atividades cotidianas e em espaços públicos, enquanto que crianças foram abusadas em ambiente doméstico; 7. a maioria das pacientes não apresentou traumas genitais ou extragenitais.

\section{Referências bibliográficas}

1. Drezett J. Estudo de fatores relacionados com a violência sexual contra crianças, adolescentes e mulheres adultas [tese]. São Paulo: Centro de Referência da Saúde da Mulher e de Nutrição, Alimentação e Desenvolvimento Infantil; 2000.

2. Kenney JW, Reinholtz C, Angelini PJ. Sexual abuse, sex before 16 , and high-risk behaviors of young females with sexually transmitted diseases. J Obstet Gynecol Neonatal Nurs 1998, 27:54-63.

3. Oliveira J. Código Penal. 25ª ed. São Paulo: Saraiva; 1987.

4. Pinho RR. Projeto de código penal: exposição de motivos. In: . Código Penal. $5^{\text {a }}$ ed., São Paulo: Atlas; 1979. p.24-66.

5. Organización Mundial de la Salud. Necesidades de salud de los adolescentes. Geneve: OMS; 1977. 28p. (Série Informes Técni$\cos , 609)$.

6. Siegel S, Castellan Jr NJ. Nonparametric statistics. $2^{\mathrm{a}}$ ed. New York: McGraw-Hill Int. Ed; 1988.

7. Hymel KP, Jenny C. Child sexual abuse. Del Med J 1997, 69:415-29.

8. Associação Brasileira Multiprofissional de Proteção à Infância e à Adolescência (ABRAPIA). Abuso sexual: mitos e realidade. Petrópolis: Autores \& Agentes Associados; 1997.

9. Beck-Sague C, Solomon F. Sexually transmitted diseases in abused children and adolescent and adult victims of rape: review of selected literature. Clin Infect Dis 1999, 1:74-83.

10. Cohen C, Matsuda NE. Crimes sexuais e sexologia forense: estudo analítico. Rev Paulista Med 1991, 109:157-64.

11. Fergusson DM, Horwood LJ, Lynskey MT. Childhood sexual abuse, adolescent sexual behaviors and sexual revictimization. Child Abuse Negl 1997, 21:789-803.

12. Hayman CR. Sexual assault on women and girls. Annals of Internal Medicine 1970, 72:277-8.

13. Holmes MM, Resnick HS, Frampton D. Follow-up of sexual assault victims. Am J Obstet Gynecol 1998, 179:336-42.

14. Massey JB, Garcia CR, Emich JP. Management of sexually assaulted females. Obstetrics and Gynecology 1971, 38:29-36.

15. Peipert JF, Domagalski LR. Epidemiology of adolescent sexual assault. Obstet Gynecol 1994, 84:867-71. 
16. Rona E, Moya G. Aspectos generales y psicopatológicos del abuso sexual en niños. Rev Chil Pediatr 1989, 60:71-5.

17. Rogers D. Physical aspects of alleged sexual assaults. Med Sci Law 1996, 36:117-22.

18. Schmuel E, Schenker JG. Violence against women: the physician's role. Eur J Obstet Gynecol Reprod Biol 1998, 80:239-45.

19. Pimentel S, Schritzmeyer ALP, Pandjiarjian V. Estupro: crime ou "cortesia"? - abordagem sociojurídica de gênero. Porto Alegre: SAFE; 1998.

20. Salvatierra SB. Incesto en adolescentes internados en el hospital psiquiatrico. Rev Costa Cienc Med 1989, 10:41-4.

21. Rickert VI, Wiemann CM. Date rape among adolescents and young adults. J Pediatr Adolesc Gynecol 1998, 11:167-75.

22. Garza-Aguilar J, Diaz-Michel E. Elementos para el estudio de la violación sexual. Salud Pública Mex 1994, 36:36-45.

23. Glaser JB, Hammerschlag MR, Mccormack WM. Epidemiology of sexually transmitted diseases in rape victims. J Infect Dis 1989, 11:246-54

24. Singh HS, Yiing WW, Nurani HN. Prevalence of childhood sexual abuse among Malaysian paramedical students. Child Abuse Negl 1996, 20:487-92.
25. Kellogg ND, Hoffman TJ. Child sexual revictimization by multiple perpetrators. Child Abuse Negl 1997, 21:953-64.

26. Belsey MA. Child abuse: measuring global problem. World Health Stat Q 1993, 46:69-77.

27. Estatuto da Criança e do Adolescente - Direitos da criança e do adolescente. São Paulo, Imprensa Oficial do Estado, 1993.

Endereço para correspondência:

Dr. Jefferson Drezett

Rua Pedroso Alvarenga 1255/64 - Itaim Bibi

CEP 04531-012 - São Paulo, SP

Fone/fax: (11) 3079.5938

E-mail: jdrezett@sti.com.br 